\title{
Strain field in dilute alloys
}

\section{PAWAN SINGH and S PRAKASH*}

Department of Physics, Centre of Advanced Study in Physics, Panjab University, Chandigarh 160014 , India

Abtract. Kanzaki lattice static method is generalized to evaluate impurity-induced strain field in the dilute alloys. The displacements of nearest neighbours of impurity are found positive and negative, however, their magnitudes are found decreasing away from the impurity.

Keywords. Hyperfine interaction; point defect; electric field gradient.

For a perfect crystal with pair potential $\phi(r)$, the total interaction energy $\Phi_{0}$ is expressed as

$$
\Phi_{0}=\sum_{n} \phi\left(\vec{R}_{n}^{0}\right),
$$

where $\vec{R}_{n}^{0}$ is the equilibrium position of the $n$th host atom. By the introduction of an impurity at the origin, the host atoms move to the new equilibrium positions $\vec{R}_{n}=\vec{R}_{n}^{0}+\vec{u}\left(\vec{R}_{n}^{0}\right)$, where $\vec{u}\left(\vec{R}_{n}^{0}\right)$ are the atomic displacements. In this configuration the total potential energy $\Phi$ in the harmonic approximation is

$$
\Phi=\Phi_{0}-\sum_{n} \sum_{\alpha} u_{\alpha}\left(\vec{R}_{n}^{0}\right) F_{\alpha}\left(\vec{R}_{n}^{0}\right)+\frac{1}{2} \sum_{n, n^{\prime}} \sum_{\alpha \beta} u_{\alpha}\left(\vec{R}_{n}^{0}\right) u_{\beta}\left(\vec{R}_{n^{\prime}}^{0}\right) \phi_{\alpha \beta}\left(n, n^{\prime}\right)
$$

where the force components

$$
F_{\alpha}\left(R_{n}^{0}\right)=-\left.\frac{\partial \phi}{\partial u_{\alpha}\left(\vec{R}_{n}^{0}\right)}\right|_{u_{\alpha}\left(\vec{R}_{n}^{0}\right)=0}
$$

and the force constants

$$
\phi_{\alpha \beta}\left(n, n^{\prime}\right)=\left.\frac{\partial^{2} \phi}{\partial u_{\alpha}\left(\vec{R}_{n}^{0}\right) \partial u_{\beta}\left(\vec{R}_{n^{\prime}}^{0}\right)}\right|_{u_{\alpha}\left(\vec{R}_{n}^{0}\right)=u_{\beta}\left(\vec{R}_{n^{\prime}}^{0}\right)=0},
$$

with $\alpha$ and $\beta(=x, y, z)$ denoting the Cartesian components and $n, n^{\prime}$, the nearest neighbours (NNs) of impurity. $F_{\alpha}\left(\vec{R}_{n}^{0}\right)$ is the force exerted on an atom at $\vec{R}_{n}^{0}$ in the $\alpha$ direction and $\phi_{\alpha \beta}\left(n, n^{\prime}\right)$ are the force constants between the atoms at $\vec{R}_{n}^{0}$ and $\vec{R}_{n^{\prime}}^{0}$ which satisfy the crystal symmetries. The equilibrium values of $u_{\alpha}\left(\vec{R}_{n}^{0}\right)$ are determined by minimizing $\Phi$ with respect to $u_{\alpha}\left(\vec{R}_{n}^{0}\right)$, which gives

$$
F_{\alpha}\left(\vec{R}_{n}^{0}\right)=\sum_{n^{\prime} \beta} \phi_{\alpha \beta}\left(n, n^{\prime}\right) u_{\beta}\left(\vec{R}_{n^{\prime}}^{0}\right) .
$$

Equation (5) shows that $u_{\alpha}\left(\vec{R}_{n}^{0}\right)$ can be evaluated if one knows $F_{\alpha}\left(\vec{R}_{n}^{0}\right)$ and $\phi_{\alpha \beta}\left(n, n^{\prime}\right)$. For computational purposes it is convenient to write (2) in Fourier space i.e.

$$
\Phi=\Phi_{0}-\sum_{\alpha \vec{q}} F_{\alpha}(\vec{q}) Q_{\alpha}(\vec{q})+\frac{N}{2} \sum_{\alpha \beta} \sum_{\vec{q}} \phi_{\alpha \beta}(\vec{q}) Q_{\alpha}(\vec{q}) Q_{\beta}(\vec{q})
$$

\footnotetext{
*Author for correspondence
} 
where

$$
\begin{aligned}
& F_{\alpha}(\vec{q})=\sum_{n} F_{\alpha}\left(\vec{R}_{n}^{0}\right) \exp \left(i \vec{q} \cdot \vec{R}_{n}^{0}\right), \\
& \phi_{\alpha \beta}(q)=\sum_{n-n^{\prime}} \phi_{\alpha \beta}\left(n-n^{\prime}\right) \exp \left[-i \vec{q} \cdot\left(\vec{R}_{n}^{0}-\vec{R}_{n^{\prime}}^{0}\right)\right],
\end{aligned}
$$

and $N$ is total number of lattice points in the crystal. $F_{\alpha}(\vec{q})$ and $\phi_{\alpha \beta}(\vec{q})$ are the Fourier transforms of $F_{\alpha}\left(\vec{R}_{n}^{0}\right)$ and $\phi_{\alpha \dot{\beta}}\left(n, n^{\prime}\right)$, respectively and $Q_{\alpha}(\vec{q})$ are the Cartesian components of normal coordinates. Minimizing (6) w.r.t. $Q_{\alpha}(\vec{q})$ etc one gets the dynamical equation

$$
\sum_{\beta}\left[N \phi_{\alpha \beta}(-\vec{q}) Q_{\beta}(\vec{q})-F_{\beta}(\vec{q}) \delta_{\alpha \beta} \delta_{-\vec{q}, \vec{q}}\right]=0 .
$$

With the knowledge of $\phi_{\alpha \beta}(\vec{q})$ and $F_{\beta}(\vec{q}),(9)$ can be solved for $\vec{Q}(\vec{q})$ which can be used to obtain $\vec{u}_{\alpha}\left(\vec{R}_{n}^{0}\right)$ by the relation

$$
\vec{u}\left(\vec{R}_{n}^{0}\right)=\sum_{q} \vec{Q}(\not{q}) \exp \left(i \vec{q} \cdot \vec{R}_{n}^{0}\right)
$$

For a central potential the dynamical matrix can be written as

$$
\phi_{\alpha \beta}(n)=\frac{R_{n \alpha}^{0} R_{n \beta}^{0}}{\left|\vec{R}_{n}^{0}\right|^{2}}\left(A_{n}-B_{n}\right)+\delta_{\alpha \beta} B_{n},
$$

where

$$
A_{n}=\left.\frac{\partial^{2} \phi}{\partial r^{2}}\right|_{r=R_{n}^{0}}, \quad B_{n}=\left.\frac{1}{\left|\vec{R}_{n}^{0}\right|} \frac{\partial \phi}{\partial r}\right|_{r=R_{n}^{0}}
$$

$R_{n \alpha}^{0}$ is the $\alpha$ Cartesian component of $\vec{R}_{n}^{0}$.

In the metallic crystals the major contribution to $\phi_{\alpha \beta}(\vec{q})$ and $F_{\alpha}(\vec{q})$ arises from the 1NN interaction due to screening. This leads to

$$
\begin{aligned}
& \phi_{\alpha \alpha}(\not)=2\left(A_{1}+B_{1}\right)\left\{2-\cos \left(q_{\alpha} \frac{a}{2}\right)\left[\cos \left(q_{\beta} \frac{a}{2}\right)+\cos \left(q_{\gamma} \frac{a}{2}\right)\right]\right\}, \\
& \phi_{\alpha \beta}(\not)=2\left(A_{1}-B_{1}\right)\left\{\sin \left(q_{\alpha} \frac{a}{2}\right) \sin \left(q_{\beta} \frac{a}{2}\right)\right\},
\end{aligned}
$$

for an fcc lattice. Here $\alpha \neq \beta \neq \gamma$ and $a$ is lattice constant. Correspondingly,

$$
F_{\alpha}(\vec{q})=i 2 \sqrt{2} F_{1} \sin \left(q_{\alpha} \frac{a}{2}\right)\left\{\cos \left(q_{\beta} \frac{a}{2}\right)+\cos \left(q_{\gamma} \frac{a}{2}\right)\right\},
$$

where $F_{\mathrm{I}}$ is the force acting at the $1 \mathrm{NN}$ site of impurity. Considering the interaction with the 2NN shell, the components of $\vec{F}(\vec{q})$ are

$$
F_{\alpha}(\bar{q})=i\left(2 F_{11}\right) \sin \left(q_{\alpha} a\right)
$$

where $F_{11}$ is the force at the $2 \mathrm{NN}$ site.

For the calculation of $\vec{F}\left(\vec{R}_{n}^{0}\right)$, we propose a general model for substitutional and interstitial impurities (Rattan et al 1993). Figure 1, part A shows the four configurations for achieving the strain field in the presence of a substitutional impurity and part 


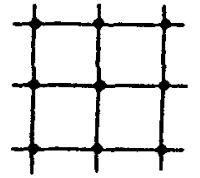

(o)

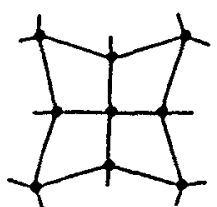

(b)

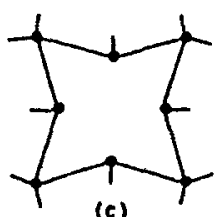

(c)

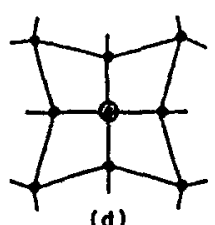

(d)

A

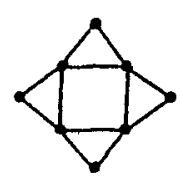

(a)

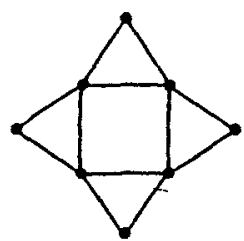

(b)

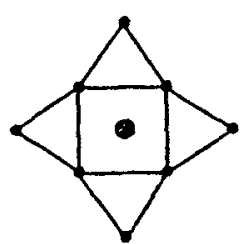

(c)

Figtre 1. A. Four configurations of the lattice for substitutional impurity and B. three configurations for an interstitial impurity.

B shows three configurations for an interstitial impurity. Let $\phi_{\mathrm{H}}(r)$ and $\phi_{\mathrm{I}}(r)$ be the host-host and impurity-host interaction potentials respectively. From figure 1 , the energy difference of configurations $(d)$ and $(a)$ is given as

$$
\begin{aligned}
\phi(d)-\phi(a)= & {[\phi(d)-\phi(c)]+[\phi(c)-\phi(b)]+[\phi(b)-\phi(a)] } \\
=\sum_{n}\left[\phi_{I}\left(\left|\vec{R}_{n}\right|\right)-\phi_{\mathrm{H}}\left(\left|\vec{R}_{n}\right|\right)\right. & \\
& \quad+\frac{1}{2} \sum_{n, n^{\prime}}\left[\phi_{\mathbf{H}}\left(\left|\vec{R}_{n^{\prime}}-\vec{R}_{n}\right|\right)-\phi_{\mathbf{H}}\left(\left|\vec{R}_{n^{\prime}}^{0}-\vec{R}_{n}^{0}\right|\right)\right] .
\end{aligned}
$$

By expanding (17) in power series of $u_{\alpha}\left(\vec{R}_{n}^{0}\right)$ and comparing with (2) it can be shown that

$$
F_{\alpha}\left(\vec{R}_{n}^{0}\right)=-\frac{\partial}{\partial u_{\alpha}\left(\vec{R}_{n}^{0}\right)} \sum_{n^{\prime}} \Delta \phi\left(\left|\vec{R}_{n^{\prime}}\right|\right)
$$

where $\Delta \phi(r)\left(=\phi_{I}(r)-\phi_{\mathbf{H}}(r)\right)$ is evaluated using dielectric screening theory (Rattan et al 1993). In this approach

$$
\Delta \phi(r)=\frac{\Delta Z Z_{\mathrm{H}} e^{2}}{|\vec{r}|^{2}}+\frac{\Omega_{0}}{\pi^{2}} \int \Delta F(q) \frac{\sin q r}{q r} q^{2} \mathrm{~d} q,
$$


Table 1. Atomic displacements (a.d.) of the NNs of $\mathrm{Mg}$ impurity in $\mathrm{Al}$ and $\mathrm{Ti}$ impurity in $\mathrm{V}$.

\begin{tabular}{lrccc}
\hline & \multicolumn{4}{c}{ Displacement components $\left(10^{-2} \mathrm{a} . \mathrm{u}.\right)$} \\
\cline { 2 - 4 } $\begin{array}{l}\text { NNs } \\
\left(n_{1}, n_{2}, n_{3}\right)\end{array}$ & \multicolumn{1}{c}{$u_{\mathrm{X}}$} & \multicolumn{1}{c}{$u_{\mathrm{Y}}$} & $u_{\mathrm{Z}}$ & $|u|$ \\
\hline $\mathrm{Al} \mathrm{Mg}$ & & & & \\
110 & -0.293 & -0.293 & 0.0 & 0.414 \\
200 & 0.433 & 0.0 & 0.0 & 0.433 \\
211 & -0.069 & -0.076 & -0.076 & 0.128 \\
220 & -0.107 & -0.107 & 0.0 & 0.151 \\
310 & 0.080 & 0.044 & 0.0 & 0.091 \\
222 & -0.056 & -0.056 & -0.056 & 0.097 \\
$\mathrm{~V} \mathrm{Ti}$ & & & & \\
111 & -0.019 & -0.019 & -0.019 & 0.033 \\
200 & 0.037 & 0.0 & 0.0 & 0.037 \\
220 & 0.010 & 0.010 & 0.0 & 0.014 \\
311 & -0.016 & 0.001 & 0.001 & 0.016 \\
222 & 0.013 & 0.013 & 0.013 & 0.023 \\
400 & 0.002 & 0.0 & 0.0 & 0.002 \\
\hline
\end{tabular}

where $\Delta Z=\left(Z_{1}-Z_{H}\right)$ is the excess impurity valency, $Z_{1}$ and $Z_{H}$ are the impurity and host valencies respectively and $\Omega_{0}$ the atomic volume. $\Delta F(q)$ is the change in the energy wave number characteristic function which is given as

$$
\Delta F(q)=\sum_{\overrightarrow{q^{\prime}}}\left(\Omega_{0} q^{2} / 8 \pi e^{2}\right)\left[V_{1}^{b}(q)-V_{\mathbf{H}}^{b}(q)\right]\left[\varepsilon_{\mathbf{H}}^{-1}\left(\vec{q}, \vec{q}^{\prime}\right)-\delta_{\vec{q}, \vec{q}}\right] V_{\mathbf{H}}^{b}\left(q^{\prime}\right) .
$$

$V_{\mathbf{H}}^{b}(\vec{q})$ and $V_{1}^{b}(\vec{q})$ are the Fourier transforms of host and impurity bare ion potentials and $\varepsilon_{\mathrm{H}}\left(\vec{q}, q^{\prime}\right)$ is the dielectric function of the host metal which is diagonal for the simple metals. Calculated $\Delta \phi(r)$ for $\mathrm{Al}(\mathrm{Mg}, \mathrm{Zn}, \mathrm{Sn})$ and $\mathrm{Cu}(\mathrm{Mg}, \mathrm{Zn}, \mathrm{Sn})$ are found oscillatory. $\Delta \phi(r)$ is found almost negligible beyond $20 \mathrm{NNs}$ of impurity.

$\Delta \phi(r)$ given in (19) is used to calculate $F_{1}$ and $F_{\text {II }}$ given in (15) and (16) with the help of (18). $A_{n}$ and $B_{n}$ are determined by numerical differentiation of $\phi_{\mathrm{H}}(r)$. These parameters determine $\phi_{\alpha \beta}(q)$ and $F_{\alpha}(q)$ defined in (13)-(16). These values are used in (9) and (10) to calculate $Q_{\beta}(\vec{q})$ and $u_{\beta}\left(\vec{R}_{n}^{0}\right)$. The results up to $6 \mathrm{NNs}$ in Al alloys are given in table 1 . These are oscillatory in nature and follow the symmetry of the lattice. Similar results are found for $\mathrm{Cu}(\mathrm{Mg}, \mathrm{Zn}, \mathrm{Sn})$.

We have also calculated the change in total potential energy of the crystal by the introduction of an impurity which is given as

$$
\Delta \phi_{\mathrm{H}}(\vec{r})=\sum_{n}^{\prime}\left[\phi_{\mathrm{H}}\left(\vec{r}-\vec{R}_{n}\right)-\phi_{\mathrm{H}}\left(\vec{r}-\vec{R}_{n}^{0}\right)\right] .
$$

A typical result for $\mathrm{Al}$ alloys along the [011] direction is shown in figure 2. $\Delta \Phi_{\mathrm{H}}(r)$ decreases rapidly as one moves away from the impurity cell. It is found to be significant up to 20 NNs. These strain fields in conjunction with valence perturbation are used to explain the EFGs (Prakash et al 1993).

Due to quasi-localized character of the conduction electrons in transition metals (TMs), $\varepsilon\left(\vec{q}, \vec{q}^{\prime}\right)$ is non-diagonal. Therefore local field effects become important. It is too difficult to calculate $\Delta \phi(r)$ with the help of $(19)$ because the non-diagonal part of $\varepsilon\left(\vec{q}, \vec{q}^{\prime}\right)$ 


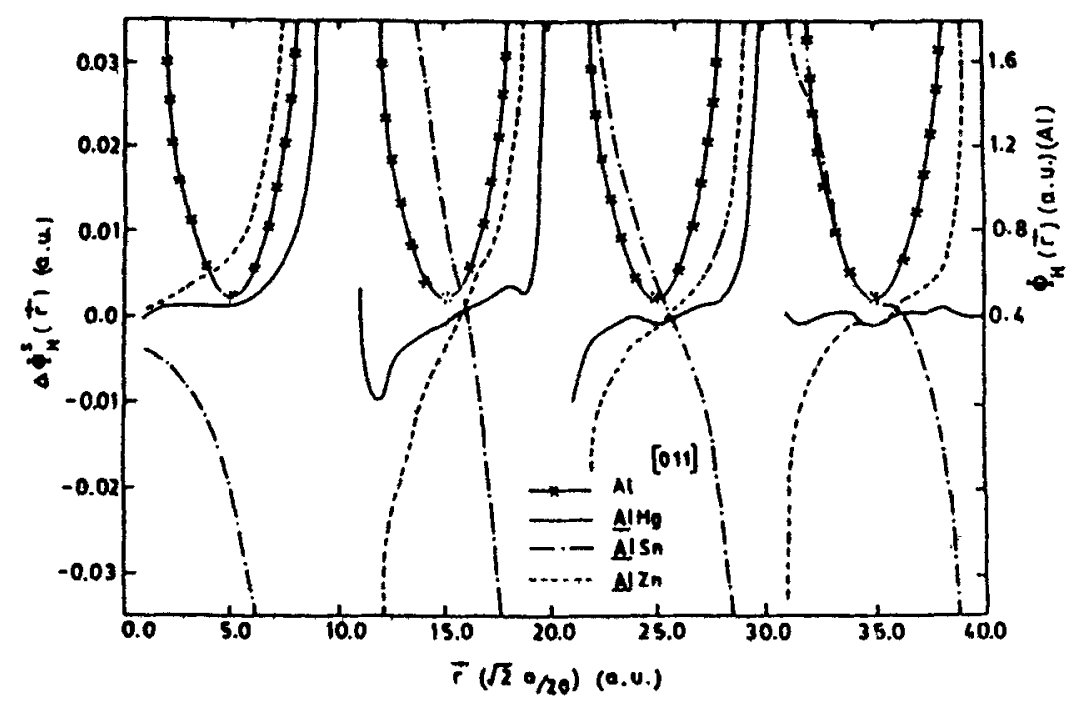

Figare 2. $\Phi_{H}(r)$ and $\Delta \Phi_{H}(r)$ vs $r$ for $A l$ and $A l$ alloys along the [011] direction.

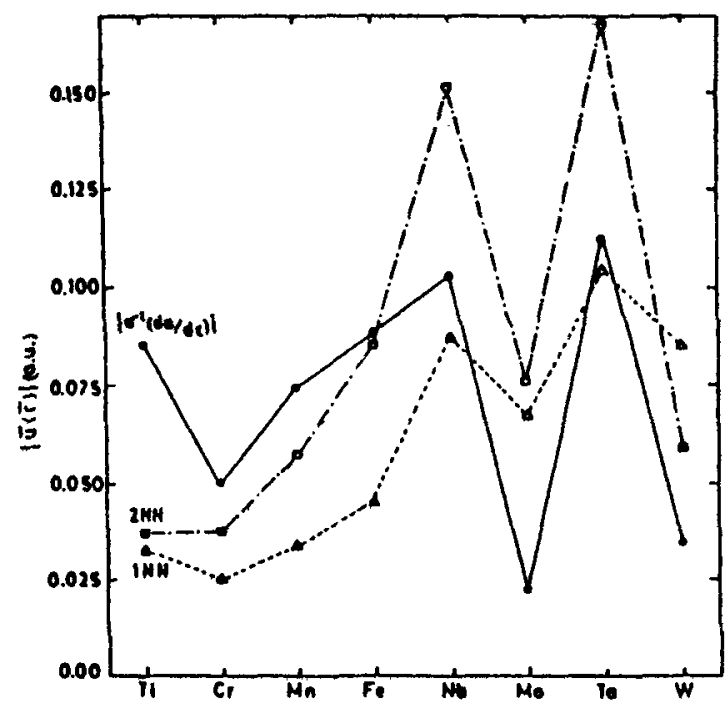

Fhare 3. The solid line represents the experimental values of fractional displacements while dotted and dashed lines represent the calculated displacements for $1 \mathrm{NN}$ and $2 \mathrm{NN}$ respectively in $\mathrm{V}(\mathrm{Ti}, \mathrm{Cr}, \mathrm{Mn}, \mathrm{Fe}, \mathrm{Nb}, \mathrm{Mo}, \mathrm{Ta}, \mathrm{W})$ alloys.

does not converge properly (Singh et al 1988). Therefore, the parameters $A_{n}, B_{n}, F_{1}$ and $F_{\mathrm{n}}$ are calculated using the following empirical potential (Wills and Harrison 1983).

where

$$
\phi_{\mathrm{H}}(r)=\phi_{\mathrm{H}}^{\mathrm{FE}}(r)+\phi_{\mathrm{H}}^{\mathrm{C}}(r)+\phi_{\mathrm{H}}^{\mathrm{b}}(r),
$$

$$
\begin{aligned}
& \phi_{\mathrm{H}}^{\mathrm{FE}}(r)=Z_{\mathrm{SH}}^{2} e^{2} \cosh ^{2}\left(\kappa r_{\mathrm{CH}}\right) \exp (-\kappa r) / r, \\
& \phi_{\mathrm{H}}^{\mathrm{C}}(r)=Z_{\mathrm{dH}}\left(225 h^{2} r_{\mathrm{dH}}^{6} / \pi^{2} m r^{8}\right), \\
& \phi_{\mathrm{H}}^{b}(r)=-Z_{\mathrm{dH}}\left(1-Z_{\mathrm{dH}} / 10\right) \sqrt{ }(12 / n)\left(28 \cdot 1 \hbar^{2} r_{\mathrm{dH}}^{3} / \pi m r^{5}\right) .
\end{aligned}
$$


Here $\phi_{\mathrm{H}}^{\mathrm{FE}}(r)$ is the free electron contribution obtained from the Ashcroft empty core model potential in conjunction with Thomas-Fermi approximation, $\kappa$ the screening length and $r_{\mathrm{CH}}$ the potential parameter. $\phi_{\mathrm{H}}^{\mathrm{C}}(r)$ is the contribution arising from the $s-d$ hybridization and depends upon $d$-state radius $r_{\mathrm{dH}}$ of the host atom. $\phi_{\mathrm{H}}^{b}(r)$ is the contribution arising from the finite $d$-band width and is of the form of a resonant bond. The number of $s$ and $d$ electrons per atom is defined such that $Z_{\mathrm{H}}=Z_{\mathrm{SH}}+Z_{\mathrm{dH}}$.

Similar potential is defined for impurity atom to calculate $\Delta \phi(r)$. Using these values of $\Delta \phi(r), u_{\alpha}\left(\vec{R}_{n}^{0}\right)$ are calculated in the same way as for simple metals. The results of atomic displacements for a particular V Ti alloy are given in table 1 (Singh et al 1994). The calculated results for atomic displacements show the same trend as found experimentally as shown in figure 3 .

\section{Acknowledgement}

One of the authors (PS) is thankful to CSIR, New Delhi for financial assistance.

\section{References}

Prakash S, Rattan S K and Singh J 1993 Phys. Rev. B48 6927

Rattan S K, Singh P, Singh J and Prakash S 1993 Phys. Rev. B47 599

Singh J, Rattan S K and Prakash S 1988 Phys. Rev. B38 10440

Singh J, Singh P, Rattan S K and Prakash S 1994 Phys. Rev. B49 932

Wills J M and Harrison W A 1983 Phys. Ret. B28 4363 